\title{
A 3-D imaging device using Compton scattering off the body
}

\author{
M. Lenti* \\ Sezione dell'INFN di Firenze, via G. Sansone 1, I-50019 Sesto F.(FI), Italy
}

Received 6 September 2007; received in revised form 1 February 2008; accepted 7 February 2008

Available online 16 February 2008

\begin{abstract}
In this paper a novel approach to 3-D imaging is discussed. A monochromatic and well collimated gamma rays beam is used to illuminate the body to be imaged; the photons Compton scattered off the body are detected by means of a Germanium strip detector which measures, with high precision, the energy of the photon and its impact position. Inverting the Compton formula, the gamma scattering position along the beam axis is calculated. The electron density of the body may be imaged with this approach. Resolutions and signal to noise ratios are discussed as a function of incident gamma ray energy; a simple simulation is presented.
\end{abstract}

(C) 2008 Elsevier B.V. All rights reserved.

PACS: 29.40.Gx; 29.40.Wk; 07.85.Fv

Keywords: Gamma-ray imaging; Compton imaging; Germanium strip detector

\section{Introduction}

3-D imaging of the human body is carried out in several ways and nuclear physics techniques have been very popular since the very beginning. $\mathrm{X}$ and gamma rays have been largely used for this purpose either using external sources (as in standard X-ray tomography or in gamma transmission tomography) or implanting radioactive nuclides in the body (as in the SPECT or in the PET case). All these methods are based on the measurement of a photon absorption profile in several directions which has to be inverted with complicated algorithms to reconstruct the desired density distribution inside the body.

Several attempts have been reported in the past to use photon Compton scattering for imaging using the energy-angle relationship [1-6]. More recently attention has been focused on reconstructing the photon arrival direction (for instance from implanted nuclides) exploiting Compton scattering in a position sensitive detector [7-9]: this method allows a conical region of possible photon origin to be defined, and with several measurements

\footnotetext{
${ }^{*}$ Tel.: + 390554572270.

E-mail address: lenti@fi.infn.it
}

the true origin can be inferred by intersection of different cones.

Here a novel method to image electron density in the body using Compton scattered gamma rays is proposed. An external gamma source is used to illuminate the body to be imaged: a well collimated and monochromatic gamma beam is prepared, the axis of which defines the line of measurement. The photons Compton scattered by electrons of the body are detected by means of a Germanium strip detector which provides the energy and the impact position of the scattered photon. Inverting the Compton formula, the scattering position along the line of measurement is reconstructed; the scattering position in the transverse direction is given by the beam axis with an uncertainty given by the beam width and divergence. In this way the electron density distribution can be determined directly without any inversion algorithm. The beam axis is later moved parallel to the previous direction and a new measurement is acquired and so on until all the desired part is imaged: this type of scanning is very flexible and for instance can be pursued with a coarse resolution (i.e. with a larger beam aperture and in a shorter time) over a relatively large area and with a good resolution in a small area in order to optimize the radiation dose. It could also be 
interesting in radiotherapy, where the same photons can be used for therapy and for imaging.

In Section 2 the main characteristics of the Compton scattering are recalled. In Section 3 the principle of the method is presented. In Section 4 a simple simulation is described where the performances of the proposed device are addressed. In Section 5 the expected resolutions are discussed while in Section 6 the background is considered; in Section 7 the fraction of detected signal events with respect to the total number of photons illuminating the body is shown.

\section{The Compton scattering}

In the Compton effect a photon of energy $E$ scatters off an electron and emerges with energy $E^{\prime}$ at an angle $\theta$ with respect to the arrival direction such that

$\cos \theta=1+\frac{m_{\mathrm{e}}}{E}-\frac{m_{\mathrm{e}}}{E^{\prime}}$

where $m_{\mathrm{e}}=511 \mathrm{keV}$ is the electron mass (natural units with speed of light $c=1$ are used) and the electron is assumed to be free and initially at rest. The kinetic energy $T$ of the knocked electron is $T=E-E^{\prime}$ and the maximum is

$T_{\max }=\frac{E}{1+m_{\mathrm{e}} / 2 E}$.

The differential cross-section per atom in the laboratory frame is given by the Klein-Nishina formula [10]:

$\frac{\mathrm{d} \sigma}{\mathrm{d} \Omega}=Z \frac{r_{\mathrm{e}}^{2}}{2}\left(\frac{E^{\prime}}{E}\right)^{2}\left[\frac{E^{\prime}}{E}+\frac{E}{E^{\prime}}-\sin ^{2} \theta\right]$

where $r_{\mathrm{e}}=2.818 \times 10^{-15} \mathrm{~m}$ is the electron classical radius and $Z$ is the atomic charge.

Electrons are bound to atoms and cannot be considered strictly at rest. This "Doppler broadening" is more important at low photon energies and depends strongly on the shell structure of the scattering atoms. Due to Doppler broadening Eq. (1) is not strictly valid but the effect can be considered as a smearing of the angular distribution of the scattering for a given initial and final photon energy. The FWHM of this smearing is tabulated for all the elements and at several photon energies [11,12] and can be introduced in the simulation; the Doppler broadening is not Gaussian but has long tails. For instance carbon has an angular broadening (FWHM) of $1.80^{\circ}$ at $200 \mathrm{keV}, 0.80^{\circ}$ at $500 \mathrm{keV}$ and $0.40^{\circ}$ at $1 \mathrm{MeV}$.

\section{The principle}

In Fig. 1 the proposed method is shown. A monochromatic gamma-emitting source illuminates the target (the body) with a well collimated gamma beam. An $x-y-z$ orthogonal coordinate system is introduced such that the $z$ axis is along the beam direction and the $x$ axis is directed towards the detector position. The gamma beam is centered along the $x=y=0$ direction. The body is

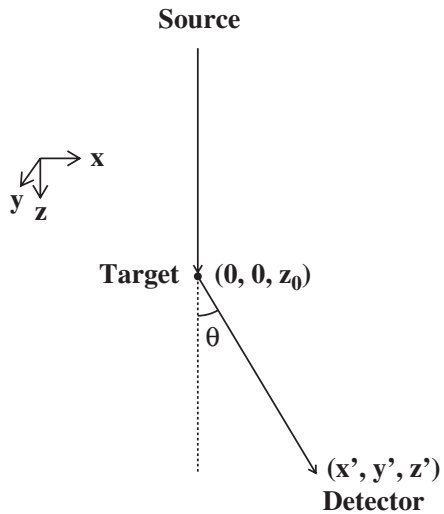

Fig. 1. A collimated gamma beam is directed towards a target and the scattered photon is observed with a Germanium strip detector. The beam is along the $z$-axis, the target is in $x=y=0$.

positioned along the beam direction in $z=z_{0}$. A photon coming from the source and scattered by the body is detected by a Germanium strip detector in the position $\left(x^{\prime}, y^{\prime}, z^{\prime}\right)$ and its energy $E^{\prime}$ is measured. Using Eq. (1) the scattering angle $\theta$ is calculated and the scattering position is reconstructed by the formula

$z_{0}=z^{\prime}-\cot \theta \sqrt{x^{\prime 2}+y^{\prime 2}}$.

\section{The simulation}

A simple simulation has been produced in order to study the performances of the apparatus. A monochromatic gamma-emitting source is placed at the beginning of a cylindrical lead collimator $400 \mathrm{~mm}$ long and $2 \mathrm{~mm}$ wide. The target (the body) is made by a cylinder $1 \mathrm{~mm}$ thick and with a $2 \mathrm{~mm}$ diameter, placed at $100 \mathrm{~mm}$ from the collimator exit with the cylinder axis parallel to the beam one; the target is made of carbon $\left(Z=6, \rho=2.265 \mathrm{~g} / \mathrm{cm}^{3}\right.$ as for graphite). A $1 \mathrm{~mm}$ thick lead absorber is placed at $300 \mathrm{~mm}$ from the target along the beam axis: it is used to stop the photons not interacted in the target but also as a source of background (see Section 6). The Germanium detector is placed at $200 \mathrm{~mm}$ from the target in a direction orthogonal to the beam axis.

One hundred million photons per gamma energy (19 different gamma energies have been used between $100 \mathrm{keV}$ and $1 \mathrm{MeV}$ with $50 \mathrm{keV}$ steps) have been generated in the gamma-emitting source with a direction pointing to the collimator exit. Interactions in a $1 \mathrm{~mm}$ thick ring around the collimator exit are taken into account. Compton scattering, Rayleigh scattering and Photoelectric absorption are simulated. The Germanium strip detector is a parallelepiped $5 \mathrm{~mm}$ thick, $100 \mathrm{~mm}$ wide and $100 \mathrm{~mm}$ high; the shortest side is parallel to the $x$ direction, the other two sides are parallel to the $y$ or $z$ directions; the center of the front face is in $x=200 \mathrm{~mm}, y=0$, $z=100 \mathrm{~mm}$. The strips on the front face are along the $z$-axis, while those on the rear face are parallel to the $y$-axis. 
The energy resolution is simulated assuming the standard $2.96 \mathrm{eV}$ energy to produce an electron-hole pair, a Fano factor of 0.05 and a constant electronics noise of $500 \mathrm{eV}$ (see for example Refs. [9,13]); the energy resolution is Gaussian. The photon impact position measurement depends on the strip pitch; $1 \mathrm{~mm}$ resolution in $z$ and $y$ is assumed (see for example Refs. [7-9] and references quoted there), while in $x$ (i.e. along the depth of the detector) the impact position is reconstructed assuming to be in the middle.

In the Germanium bulk Photoelectric absorption and Compton scattering are simulated up to a maximum of two interactions overall (in the collimator exit, in the target and in the absorber only one interaction, or none, is simulated). The $11 \mathrm{keV}$ Germanium $\mathrm{K}_{\alpha} \mathrm{X}$-ray escape peak is neglected. In case of two interactions the total reconstructed deposited energy is given by the sum of the two deposits, while the reconstructed impact position is the average of the two interactions positions weighted with the deposited energies, if the distance between them is smaller than five standard deviations, or is the position of the first interaction if the distance is larger.

In the simulation the Compton scattering is generated using Eq. (3), the Rayleigh scattering and the Photoelectric effect are generated by interpolation of data coming from Ref. [14]. The Doppler broadening in the Compton scattering is generated in a Gaussian way assuming the FWHM tabulated in Ref. [11]; neglecting the non-Gaussian tails is not a large effect in this case (small scattering body) but can underestimate the difficulties in imaging extended objects (not considered here).

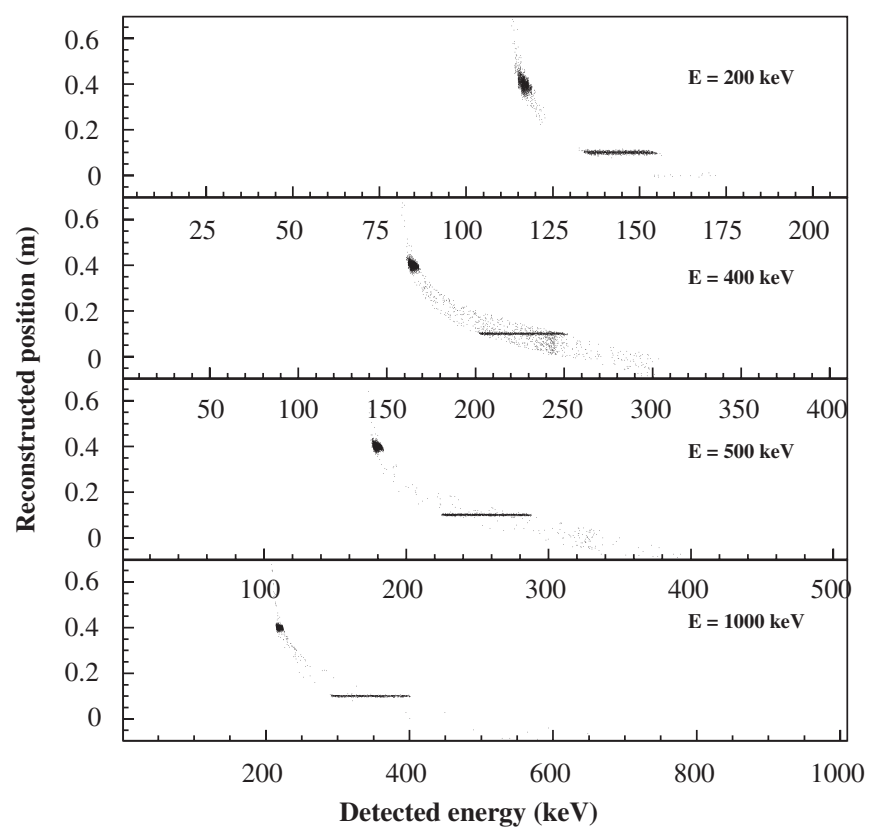

Fig. 2. Reconstructed scattering position versus deposited energy on and measured by the Germanium strip detector by photons of four different initial energies. The horizontal scales of the four plots are different, the vertical scales are the same.
In Fig. 2 the reconstructed scattering position $z_{0}$ versus the deposited energy is shown for four different gamma initial energies. The thin horizontal band at $z_{0}=0.1 \mathrm{~m}$ is the target image, the broad band around $z_{0}=0.4 \mathrm{~m}$ is the absorber image and around $z_{0}=0$ is hardly visible the collimator image. Some relevant features are clearly visible: at $400 \mathrm{keV}$ initial energy the background level is much higher than at $200 \mathrm{keV}$ or at $500 \mathrm{keV}$; the $z_{0}$ resolution improves at higher initial energy, while the number of detected events decreases.

In Fig. 3 the deposited energy distribution is shown for the same four different gamma initial energies. The full energy peak of Rayleigh scattered photons is visible on the extreme right. A well characterized band, lightly shaded in the plot, is the full energy deposit of photons Compton scattered by the target. The full energy peak of photons Compton scattered by the absorber is clearly visible. The rest of the events are due to photons escaping from the detector after one or two Compton scatters in it. When the tail of Compton scattering in the detector overlaps with the full energy deposit of photons Compton scattered by the target, a background builds up.

The signal is selected requiring that the deposited energy is compatible with a photon Compton scattered at $\theta=$ $90^{\circ} \pm 15^{\circ}$ (see Section 5). In Fig. 4 the photon scattering reconstructed position is shown for all the events; the lightly shaded histogram is for photons coming from Compton scattering in the target, the heavily shaded histogram for events passing the described cut but not

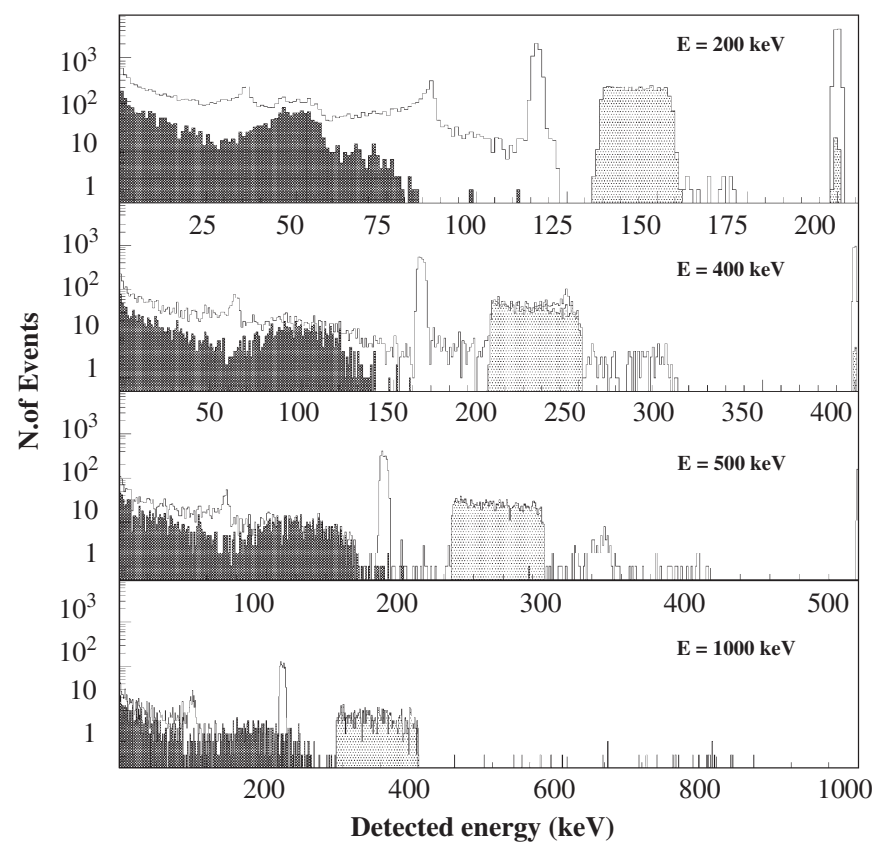

Fig. 3. Energy deposited on and measured by the Germanium strip detector by scattered photons of four different initial energies. The horizontal scales of the four plots are different, the vertical scales are logarithmic and are the same. Photons scattered off the body are shown in the lightly shaded histogram if fully absorbed by the detector and in the heavily shaded histogram if escaping the detector after one or two interactions. 


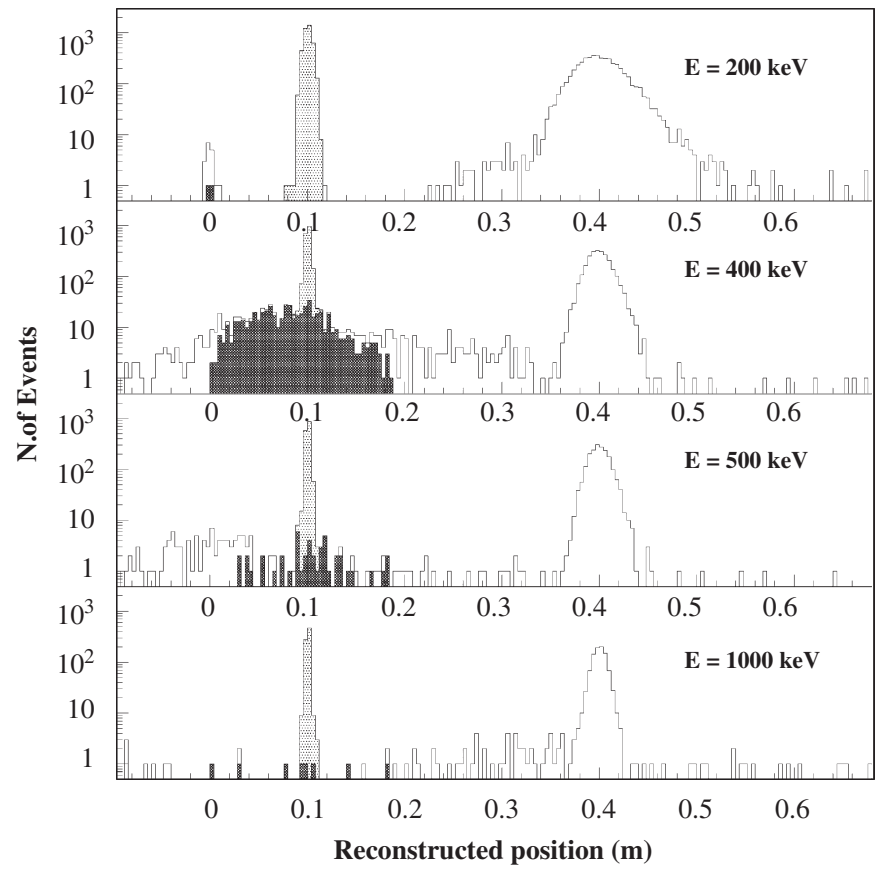

Fig. 4. Reconstructed position along the beam axis of the photon scattering point for four different initial energies. The horizontal and vertical scales are the same for the four plots, the vertical scales are logarithmic. Events with the energy cut described in the text are shown in the lightly shaded histogram if coming from scattering off the body, in the heavily shaded histogram otherwise.

coming from the target. Note that most of the photons not fully absorbed in the detector, visible in Fig. 3, are reconstructed at large and negative $z_{0}$ or not reconstructed at all and are not visible in Figs. 4 and 2. Resolution and background can be easily estimated and will be addressed in the following sections.

\section{The resolution}

From Eq. (4) it is clear that the best $z_{0}$ resolution is achieved at $\theta=90^{\circ}$. Calling $d=\sqrt{x^{\prime 2}+y^{\prime 2}}$ the distance of the photon impact point on the detector from the beam axis, the resolution on $z_{0}$ (without the contribution from the beam divergence and width, shown later) can be found as

$$
\begin{aligned}
\Delta z_{0}= & \Delta z^{\prime} \oplus d \Delta \theta_{\text {Doppler }} \oplus d \frac{m_{\mathrm{e}}}{E^{\prime}}\left(\frac{\Delta E^{\prime}}{E^{\prime}}\right) \oplus \overline{|\cot \theta|} \\
& \times\left(\frac{x^{\prime}}{d} \Delta x^{\prime} \oplus \frac{y^{\prime}}{d} \Delta y^{\prime}\right)
\end{aligned}
$$

where $\oplus$ means sum in quadrature. In Eq. (5) $\Delta z^{\prime}$ is the resolution on the photon impact position along the $z$ axis given by the strip granularity; $\Delta \theta_{\text {Doppler }}$ is the contribution from the Doppler broadening to the Compton scattering; $\Delta E^{\prime} / E^{\prime}$ is the energy resolution of the Germanium detector. $\Delta x^{\prime}$ and $\Delta y^{\prime}$ depend on the Germanium detector thickness and strip pitch, respectively, but their contribution is suppressed by a factor $\overline{|\cot \theta|}$ which is 0 if only $\theta=90^{\circ}$ is accepted. $\overline{|\cot \theta|}$ is an average over the accepted $\theta$ range

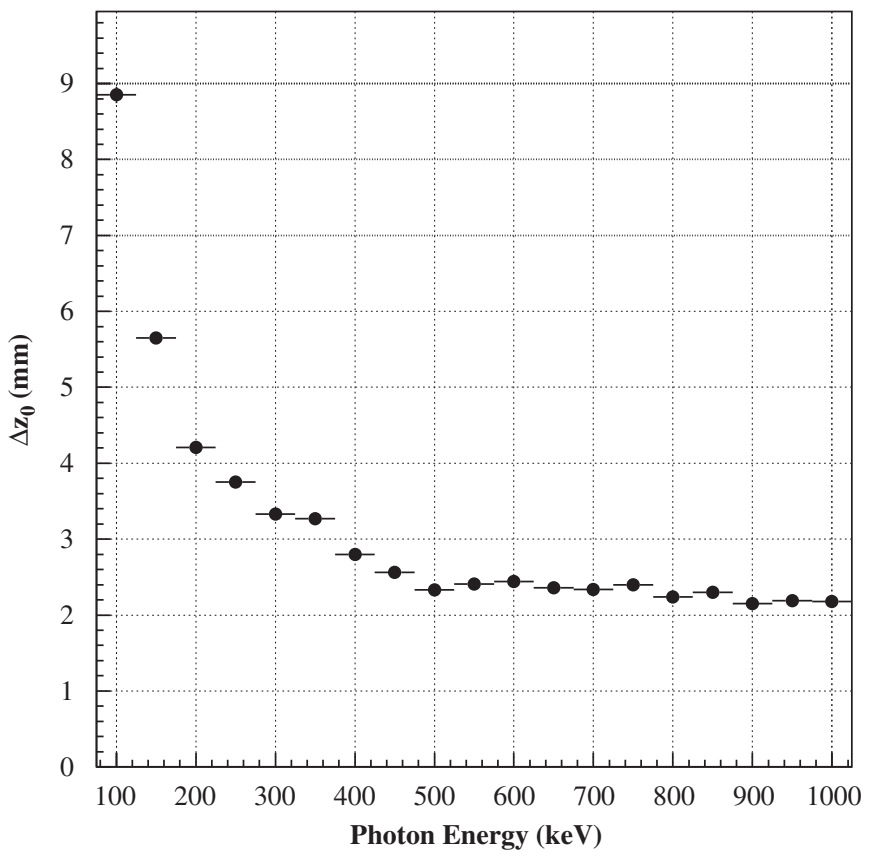

Fig. 5. Resolution (r.m.s.) of the reconstructed position of Compton scattering along the line of measurement (i.e. the collimated gamma rays beam) as a function of the gamma initial energy as derived by the simulation described in the text.

(about 0.13 for $\theta=90^{\circ} \pm 15^{\circ}$ ). The error induced by the beam divergence $\Delta \theta_{\text {beam }}$ and width $\Delta w_{\text {beam }}$ turns out to be

$\Delta z_{0}($ beam $)=d \Delta \theta_{\text {beam }} \oplus \overline{|\cot \theta|} \Delta w_{\text {beam }}$

where the first term is the dominant one. The finite thickness of the target also contributes to the total resolution.

The $z_{0}$ resolution improves as $d$ decreases, i.e. the detector must be as close as possible to the body. The $z_{0}$ resolution also improves if the gamma energy increases, both because the energy resolution improves and because the Doppler broadening decreases. As it will be shown later, the number of scattered and detected photons decreases as $E$ increases and also the background changes. The best gamma energy is thus a compromise among different aspects.

It is worth noting that the $z_{0}$ resolution is only slightly sensitive to $\Delta x^{\prime}$ and $\Delta y^{\prime}$.

In Fig. 5 the reconstructed scattering position resolution (r.m.s.) is shown as a function of the gamma initial energy; above $500 \mathrm{keV}$ no further improvement can be seen. The reconstructed position resolution at low gamma initial energy is dominated by the energy resolution and by the Doppler broadening; at high gamma initial energy the dominant contributions are due to the effect of multiple interactions in the detector, followed by the $z^{\prime}$ resolution, then the Doppler broadening and the energy resolution (the finite size of the target gives a smaller contribution). 


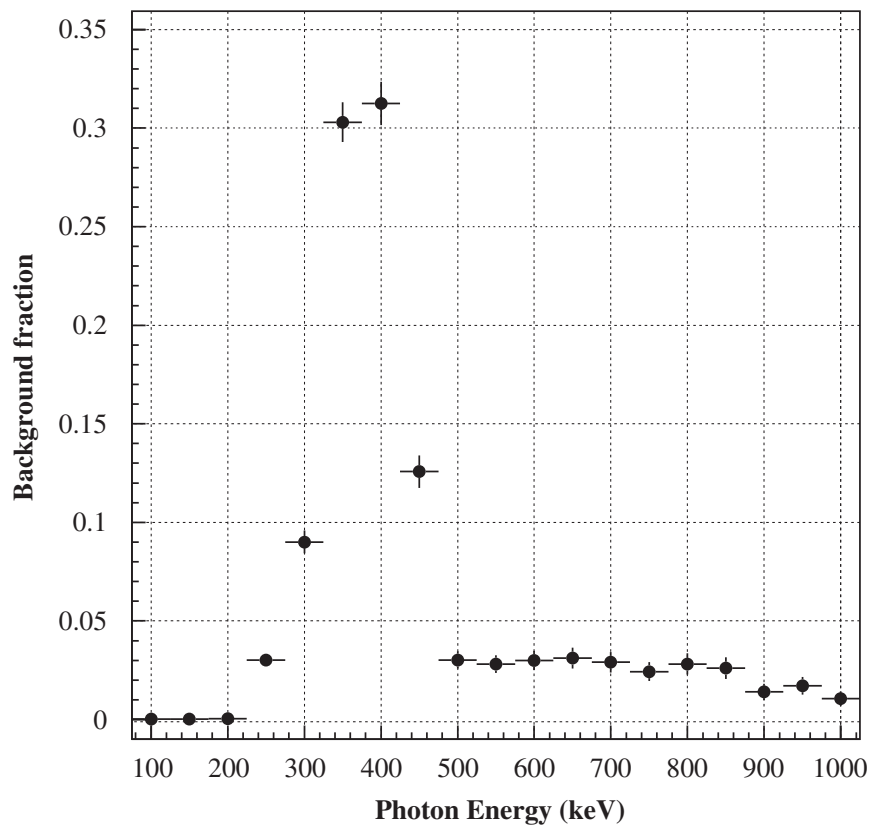

Fig. 6. Background fraction as a function of the gamma initial energy as derived by the simulation described in the text.

\section{The background}

The signal is made by photons Compton scattered by the body and fully absorbed by the detector. The background induced by the source is made by photons scattered (Compton or Rayleigh) by the collimator or the absorber and not fully absorbed by the detector (i.e. escaping the detector after one or more Compton scattering in it). When the detector Compton edge relative to the photon initial energy (i.e. after a Rayleigh scattering) falls inside the accepted energy range for the signal, a clear background is built up. From Eq. (2) and assuming an accepted energy range corresponding to Compton scattering off the body with $\theta=90^{\circ} \pm 15^{\circ}$, the initial energy to be avoided is between 322 and $420 \mathrm{keV}$. Taking into account the tails, two region of gamma energy are acceptable: one below $250 \mathrm{keV}$ and one above $500 \mathrm{keV}$.

In Fig. 6 the background fraction as a function of the gamma initial energy is shown. The net increase of background above 250 and below $500 \mathrm{keV}$ is clearly visible.

\section{The number of signal events}

The number of signal events decreases as a function of the gamma initial energy because the Compton crosssection at $\theta=90^{\circ}$ decreases and because the efficiency of the Germanium strip detector also decreases. In Fig. 7 the number of signal events detected with the above described cut is shown as a function of the gamma initial energy for the illumination discussed in Section 4. Between 250 and $500 \mathrm{keV}$ (the borders of the background peak discussed in Section 6) the number of signal events drops by about a factor of two.

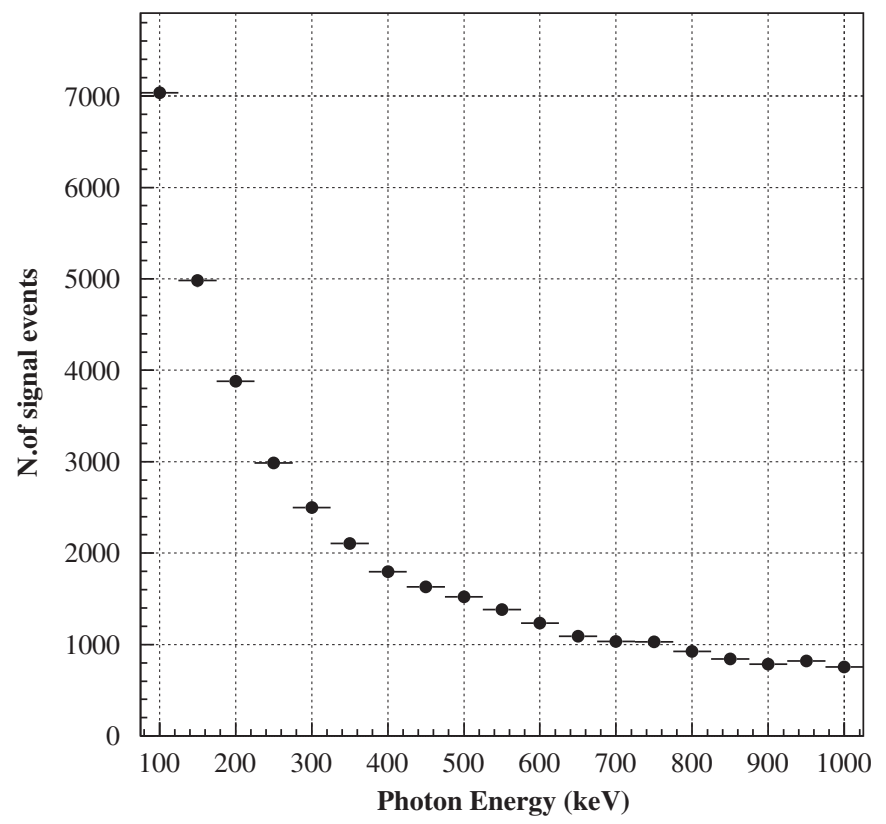

Fig. 7. Number of signal events as a function of the gamma initial energy as derived by the simulation described in the text.

The number of detected signal events is a few thousands starting from 100 million generated photons exiting from the collimator. The target seen from the collimator exit has an acceptance of $64 \%$. Assuming a gamma initial energy of $500 \mathrm{keV}$ the Compton scattering probability in the target is $2 \%$, the probability that the scattering takes place at $\theta=$ $90^{\circ} \pm 15^{\circ}$ is about $8 \%$ and that the scattered photon crosses the detector $100 \mathrm{~mm}$ wide at $200 \mathrm{~mm}$ distance is about $8 \%$; finally the probability that the photon interacts in the detector is $36 \%$ and then that it deposits all its energy is about $50 \%$. The total probability to collect a signal event at $500 \mathrm{keV}$ gamma initial energy with the described setup, for a photon coming from the collimator exit, is about $1.5 \times 10^{-5}$. The number of collected signal events can be made larger by increasing the azimuthal angle coverage of the detector (i.e. using a larger detector or more detectors at the same distance from the beam axis), while an increase on the $\theta$ coverage brings a deterioration of the resolution performances. An increase in the detector thickness allows collection of more signal events but the $z_{0}$ resolution gets worse because the fraction of multiple interactions in the detector becomes larger.

\section{Conclusions}

In this paper a novel method of 3-D imaging has been presented. The very good performances of a Germanium strip detector are used to determine the Compton scattering positions of photons coming from a monochromatic and well collimated gamma beam, measuring the electron density distribution of the scattering body with resolutions approaching $2 \mathrm{~mm}$. Gamma initial energies of about $250 \mathrm{keV}$ (which provide larger statistics) or $500 \mathrm{keV}$ 
(which guarantee a better position resolution) appear the most reasonable choices. A simple simulation with a graphite target has been discussed in detail and from which one can extrapolate the performances of the method to other geometries and materials.

\section{References}

[1] G. Harding, J. Kosanetzky, Nucl. Instr. and Meth. 280 (1989) 517.

[2] T.H. Prettyman, R.P. Gardner, J.C. Russ, K. Verghese, Appl. Radiat. Isot. 44 (1993) 1327.

[3] N.V. Arendtsz, E.M.A. Hussein, IEEE Trans. Nucl. Sci. NS-42 (1995) 2155.

[4] S.J. Norton, J. Appl. Phys. 76 (1994) 2007.

[5] B.L. Evans, J.B. Martin, L.W. Burggraf, M.C. Roggemann, T.N. Hangartner, Nucl. Instr. and Meth. A 480 (2002) 797.

[6] L. Lawson, Mater. Eval. 53 (1995) 936.
[7] E.A. Wulf, B.F. Phlips, W.N. Johnson, R.A. Kroeger, J.D. Kurfess, IEEE Trans. Nucl. Sci. NS-50 (4) (2003) 1182.

[8] C.J. Hall, W.I. Helsby, R.A. Lewis, P. Nolan, A. Boston, Nucl. Instr. and Meth. A 513 (2003) 47.

[9] J. van der Marel, B. Cederwall, Nucl. Instr. and Meth. A 471 (2001) 276.

[10] J.D. Bjorken, S.D. Drell, Relativistic Quantum Mechanics, McGrawHill, New York, 1964.

[11] A. Zoglauer, G. Kanbach, Doppler broadening as a lower limit to the angular resolution of next generation compton telescope, in: Proceedings of the 2002 Astronomical Telescopes and Instrumentation, SPIE, 2002.

[12] C. Ordonez, A. Bolozdynya, W. Chang, Doppler broadening of energy spectra in compton camera, in: Proceedings of the 1997 IEEE Nuclear Science Symposium, vol. 2, 1998, pp. 1361-1365.

[13] G.F. Knoll, Radiation Detection and Measurement, second ed., Wiley, New York, 1989.

[14] 〈http://physics.nist.gov/PhysRefData〉. 